\title{
Features of deformation and breaking for Ni nanowire
}

\author{
M. D. Starostenkov ${ }^{1 \dagger}$, M. M. Aish ${ }^{1,2}$ \\ ${ }^{1}$ Altai State Technical University, Lenina Pr. 46, 656038, Barnaul, Russia \\ ${ }^{2}$ Menouia university, Gamal Abdel Nasser St., Shebin El-Kom, Al-Minufya, Egypt \\ †genphys@mail.ru
}

Molecular dynamics (MD) simulations have been carried out on pure Nickel (Ni) crystal with face-centered cubic (FCC) lattice upon application of uniaxial tension at nanolevel with a speed of $20 \mathrm{~m} / \mathrm{s}$. The deformation corresponds to the direction $\langle 001\rangle$. To the calculated block of crystal free boundary conditions are applied in the directions $\langle 100\rangle,\langle 010\rangle$. A manybody interatomic potential for $\mathrm{Ni}$ within the second-moment approximation of the tight-binding model (the Cleri and Rosato potentials) was employed to carry out three dimensional MD simulations. A computer experiment is performed at a temperature corresponding to $10 \mathrm{~K}, 300 \mathrm{~K}$ and $1000 \mathrm{~K}$. MD simulation used to investigate the effect of length of Ni nanowire on the nature of deformation and fracture. The feature of deformation energy can be divided into four regions: quasi-elastic, plastic, flow and failure. The nature of deformation, slipping, twinning and necking were studied. Stress decreased with increasing volume. The results showed that breaking position depended on the nanowire length.

Keywords: deformation, fracture, molecular dynamics, nanowire.

\section{Introduction}

The description of interactions between atoms is very crucial in any atomistic simulation, ranging from first-principles electronic structure calculations to semi-empirical methods. $\mathrm{Ab}$-initio techniques are superior, accurate and can supply information on diffusion barriers, but they are limited by high computational costs. They are therefore used for relatively short time scales and small systems. In order to simulate longer time scales and larger systems parameter-based empirical methods such as the many-body potentials of the embedded-atom method (EAM) [1,2], Finnis - Sinclair [3] potentials and the second-moment approximation (SMA) of the tight-binding (TB) method [4-9] are found to be very efficient. The semi-empirical methods, although less accurate, are very fast and able to simulate the dynamical character of diffusion and reveal different elementary processes (e.g., hopping or adatom-surface exchange). In particular, the TB-SMA expression [7-9] of the total energy of a metallic system is based on a small set of adjustable parameters, which can be determined by adjusting to experimental data $[9,10]$ or ab-initio results $[1,10,11]$.

The aim of the present work is to use this interatomic potential with molecular-dynamics (MD) simulations to study the mechanical properties of Ni nanowire.

\section{Molecular dynamics simulations}

The interatomic interactions were calculated using the tightbinding potentials [12]. We used a simpler, more efficient computer program for simulating a system involving several thousands of atoms in the time interval up to several nanoseconds. It should be noted that, even in the current state of the art in the development of high-performance computers, an ab-initio simulation of this system remains impossible. On the other hand, the potentials proposed by
Cleri and Rosato [12] have already worked well in cluster studies [13-19].

In our model, the potential energy of the system was calculated from the relationship

$$
U=\sum_{i}\left(E_{b}^{i}+E_{r}^{i}\right)
$$

where

$$
E_{r}^{i}=\sum_{j} A \exp \left[-p\left(\frac{r_{i j}}{r_{0}}-1\right)\right]
$$

is the two-body term, and

$$
\begin{gathered}
E_{b}^{l}=-\sqrt{\rho_{i}} \\
\rho_{i}=\sum_{j \neq 1} \zeta^{2} \exp \left[2 q\left(\frac{r_{i j}}{r_{0}}-1\right)\right]
\end{gathered}
$$

is the many-body term.

Where $r_{0}$ is the equilibrium distance between atoms, rij is the distance between the $i^{\text {th }}$ and $j^{\text {th }}$ atoms, and $A, \xi, p$ and $q$ are fitted parameters these parameters were taken directly from [12].

Despite its simple functional form, the model can be used to describe quite well elastic, defect and melting properties of a wide range of FCC- and HCP-metals. The velocities of atomic motion in the simulation were determined using the Verlet algorithm with a time step $h=2 \mathrm{fs}$. The potentials proposed by Cleri and Rosato [12] have already worked well in cluster studies.

The initial velocities of particles are a Maxwell-Boltzmann distribution corresponding to a given temperature. They are given by

$$
\frac{N(v)}{N}=\sqrt{\frac{m}{2 \Pi k T}} \exp \left(-\frac{m v^{2}}{2 k T}\right)
$$



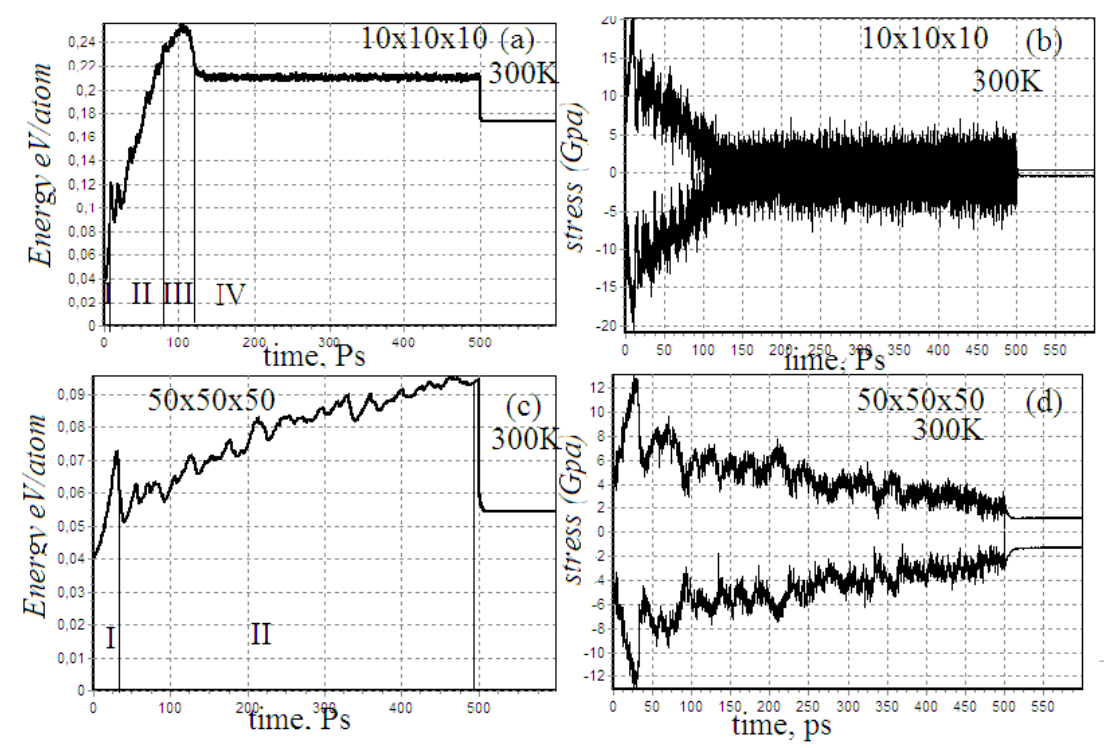

Fig. 1. Dependence of the stored energy of deformation of the experiment at 300K (a) for 10x10x10, (b) 50x50x50 Ni nanowires and stress with time (c) for 10x10x10, (d) 50x50x50.

where $N(v)$ denotes the number of particles which have velocity $v ; k$ is the Boltzmann's constant, and $T$ is the temperature. To keep the system temperature, the following correction is required:

$$
v_{i}^{\text {final }}=v_{i} \sqrt{\frac{T_{d}}{T_{a}}},
$$

where is the velocity of the particle $i$ after correction. $T_{d}$ and $T_{a}$ are the desired temperature and actual temperature of system, respectively.

The object of investigation is different nanowires of $\mathrm{Ni}$ alloy. Alloy structure is presented in the form of a face-centered cubic cell. We study the effect of volume on the mechanical properties of simulation nanowires at the temperatures $300 \mathrm{~K}$ and $1000 \mathrm{~K}[15,20-23]$. The estimated size of the crystal unit was from 163 atoms ( 5 atoms along the edges at the bottom and 5 in height) to 72500 atoms (50 atoms along the edges at the bottom and 50 in height) for various experiments.

Table1.

\begin{tabular}{|c|c|c|c|c|c|}
\hline & system & $\mathrm{f}_{0}(\mathrm{~nm})$ & $\mathrm{t}_{\mathrm{pl}}(\mathrm{ps})$ & $\begin{array}{c}\sigma_{\mathrm{T}} \\
(\mathrm{GPa})\end{array}$ & $\mathrm{t}_{\mathrm{b}}(\mathrm{ps})$ \\
\hline 1 & $5 \times 5 \times 5$ & 2.3 & 6 & 30 & 49 \\
\hline 2 & $10 \times 10 \times 10$ & 4.5 & 10 & 20 & 115 \\
\hline 3 & $20 \times 20 \times 20$ & 9.1 & 19 & 18 & 300 \\
\hline 4 & $24 \times 24 \times 24$ & 10.1 & 23 & 16 & 305 \\
\hline 5 & $30 \times 30 \times 30$ & 14.2 & 25 & 15 & 430 \\
\hline 6 & $36 \times 36 \times 36$ & 16.7 & 27 & 14 & 475 \\
\hline 7 & $40 \times 40 \times 40$ & 19 & 27 & 13 & 550 \\
\hline 8 & $50 \times 50 \times 50$ & 21.6 & 30 & 12 & - \\
\hline
\end{tabular}

Table 2.

\begin{tabular}{|c|c|c|c|c|c|}
\hline & system & $\mathrm{I}_{0}(\mathrm{~nm})$ & $\mathrm{t}_{\mathrm{pl}}(\mathrm{ps})$ & $\begin{array}{c}\sigma_{\mathrm{T}} \\
(\mathrm{GPa})\end{array}$ & $\mathrm{t}_{\mathrm{b}}(\mathrm{ps})$ \\
\hline 1 & $5 \times 5 \times 5$ & 2.3 & 6 & 22 & 39 \\
\hline 2 & $10 \times 10 \times 10$ & 4.5 & 10 & 16 & 100 \\
\hline 3 & $20 \times 20 \times 20$ & 9.1 & 19 & 13 & 270 \\
\hline 4 & $24 \times 24 \times 24$ & 10.1 & 23 & 12 & 243 \\
\hline 5 & $30 \times 30 \times 30$ & 14.2 & 25 & 11 & 396 \\
\hline 6 & $36 \times 36 \times 36$ & 16.7 & 27 & 10 & 450 \\
\hline 7 & $40 \times 40 \times 40$ & 19 & 27 & 8 & 520 \\
\hline 8 & $50 \times 50 \times 50$ & 21.6 & 30 & 7 & - \\
\hline
\end{tabular}

Fig. 2. Simulated ultimate strength of ultrathin $\mathrm{Ni}$ nanowires as a function of nanowire length for different temperatures.

Since the breaking and the Yielding of $\mathrm{Ni}$ nanowires are of main interest in this work (tab.1 and 2), it seems to be reasonable to adopt small $L_{x} L_{y}$ and $L_{z}$ for the simulations. To save the computing time, the dimensions of the MD models used in the following simulations are set to be $L_{x}=L_{y}=L_{z}$ and changes from 5 to 50 . The nature of deformation, slipping, twinning and necking was studied.

MD calculation results for nanoblocks of different sizes at $300 \mathrm{~K}$, depending on the the initial length $\left(l_{0}\right)$, the start of plastic deformation $\left(t_{p l}\right)$, yield strength $\left(\sigma_{T}\right)$, and destruction of the sample start time $\left(t_{b}\right)$ were carried out.

\subsection{Four stages deformation for different $\mathrm{Ni}$ nanowires}

The plots of the stored energy of deformation with the time, reflecting the processes in the nanowire during deformation were obtained (fig.1).

There are four stages of deformation: the quasi-elastic deformation (I), plastic deformation (II), the breaking (flow) (III), and failure (IV). At all volumes, in the first stage there was almost linear increase in stress. The initial stage quasielastic area there is only relative displacement of atoms and there are no defects. Therefore, in this region the energy

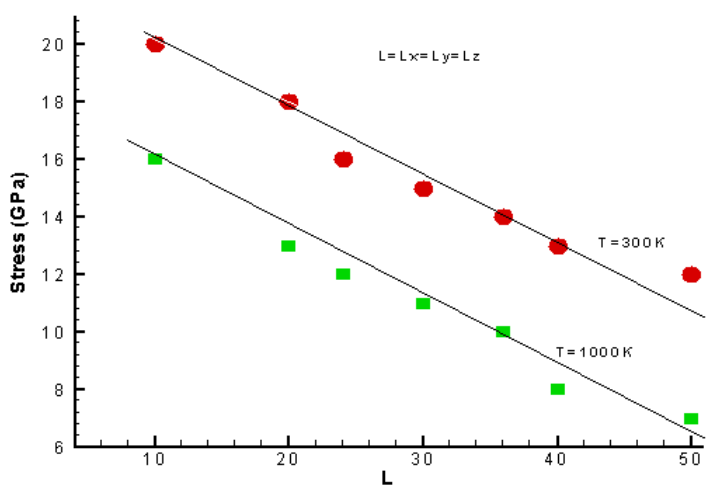



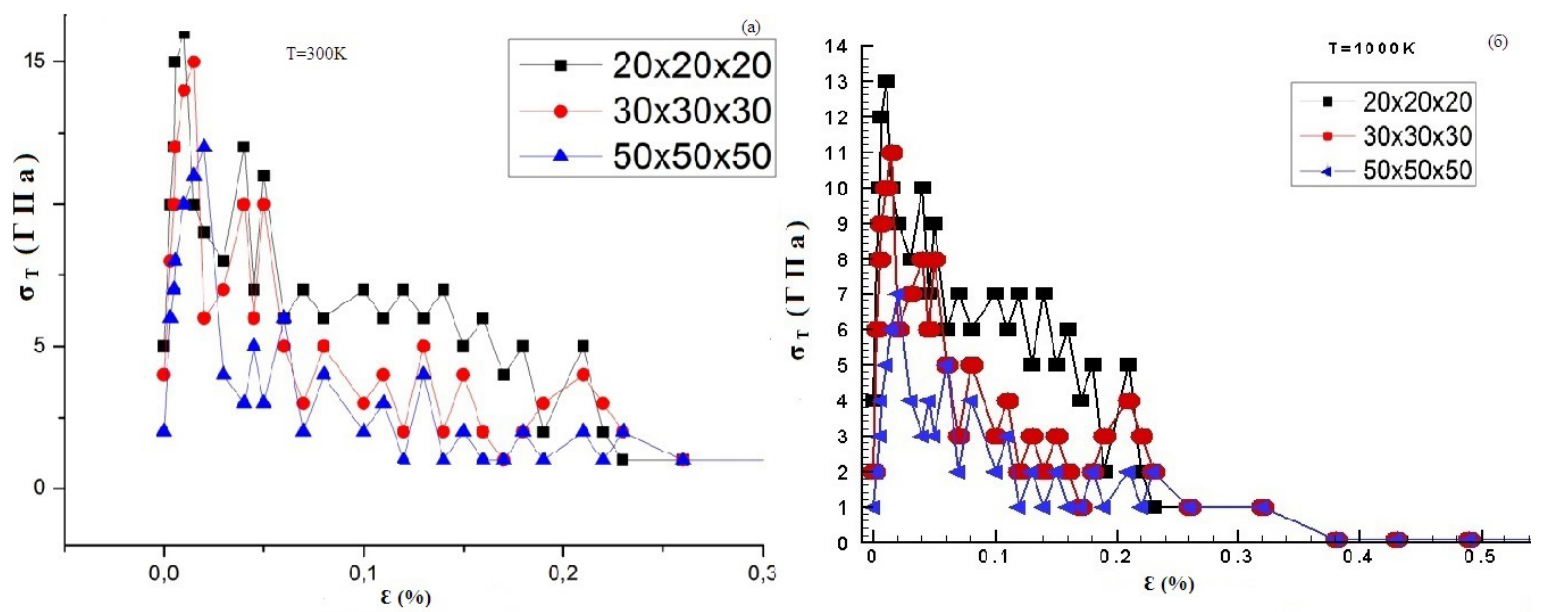

Fig. 3. Relationship between tensile stress of different Ni nanowires with tensile strain at (a) $300 \mathrm{~K}$ and (b) $1000 \mathrm{~K}$.

stored varies periodically. This stage is completed in $6 \mathrm{ps}$ for

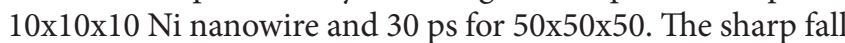
takes place only at the point of transition from the first to second stages of deformation (fig.1a and c).

Experiments have shown that when the volume increases the first and second stages of deformation were widened. The neck of the nanowire forms after the slips happened, and the deformations have been carried mainly through the elongation of the neck. Beyond the neck region, atomic structures have no significant changes. The atomic rearrangements in the neck region induce the zigzag increase-decrease in stress as the strain is increased. The atoms, close to the narrowest region of the neck, are highly disordered. At the point of breaking, we observe a one-atom thick layer. With further pulling of the nanowire, the bond between the two atoms lying in the one-atom breaks and then the rupture happened.

\subsection{Volume effect on stress}

Figure 2 shows the stress-length relations obtained from the simulations for temperature at $300 \mathrm{~K}$ as listed in tab.1. As can be seen from the figure, the simulated stress decreased with increasing volume. However, further analysis for high temperature at $1000 \mathrm{~K}$ (tab.2) shows that the stress-length curves exhibit a large oscillation about a mean curve (fig.2); however, the magnitudes of the oscillation can be reduced with increasing number of atoms.

\subsection{Stress strain relation for different $\mathrm{Ni}$ nanowires}

With MD simulations, the uniaxial tension of the nickel nanowires are studied of different nanowire volume at $300 \mathrm{~K}$ and $1000 \mathrm{~K}$. Figure 3 gives the stress-strain relationships for the $20 \times 20 \times 20,30 \times 30 \times 30$ and $50 \times 50 \times 50 \mathrm{Ni}$ nanowires subjected to uniaxial tension at temperatures $300 \mathrm{~K}$.

\subsection{Effect of volume on the breaking position and time}

Figure 4 presents the breaking for $\mathrm{Ni}$ nanowires. The breaking position depends on nanowire volume. Surface atoms play an important role in the mechanical behaviors
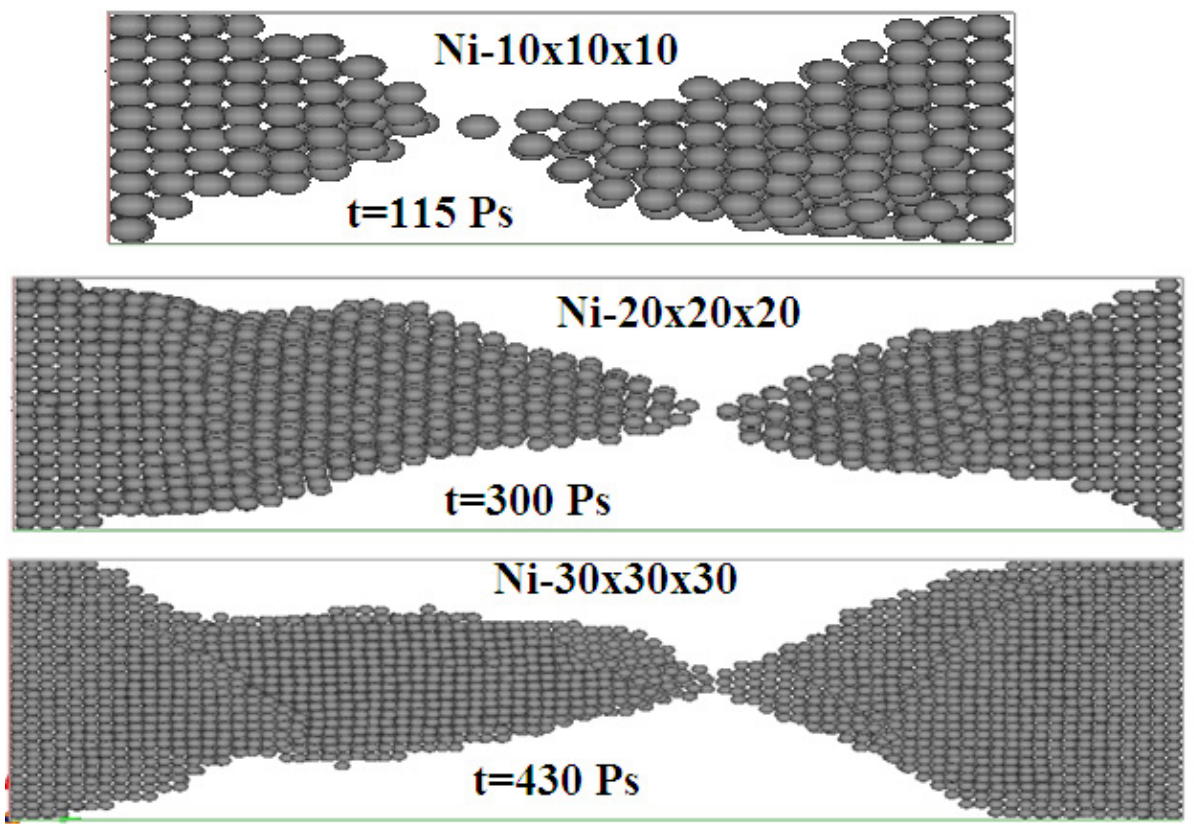

Fig. 4. Snapshots of Ni nanowires with different volume at the breaking moment. 
of nano structures, and the volume effect commonly found in small-scale systems is the surface effect. If the breaking position is predictable, the nanowire can be strengthened near the breaking position to avoid failure. Although the single breaking case is not predictable, many breaking cases show a statistic feature. Figure 4 presents the representative snapshots of $\mathrm{Ni}$ nanowires with different Volume at the breaking moment. In most cases, the final breaking position occurs at the central part of the nanowire when it is short, as the nanowire length increases the breaking position gradually shifts to the ends.

\section{Conclusions}

A many-body interatomic potential for $\mathrm{Ni}$ within the second-moment approximation of the tight-binding model (the Cleri and Rosato potentials) was employed to carry out three dimensional MD simulations. MD simulation results for $\mathrm{Ni}$ nanowire at the temperatures $300 \mathrm{~K}$ and $1000 \mathrm{~K}$ are presented. The mechanical properties for these temperatures are different. The stress-time and stress-length curves for nanowires are shown [22-27]. The breaking and yield stress of nanowires are dependent on the volume and temperature. The necking, plastic deformation, slipping domain, twins, clusters, microspores and break-up phenomena of nanowire are demonstrated. Stress decreases with increasing nanowire volume and temperature. The final breaking position occurs at the central part of the nanowire when it is short. As the nanowire length increases the breaking position gradually shifts to the ends.

\section{References}

1. F. Ercolessi, J. B. Adams. Europhys. Lett. 26, 583 (1994).

2. Y. Mishin, D. Farkas, M.J. Mehl and D. A. Papaconstantopoulos. Phys. Rev. B. 59, 3393 (1999).

3. Y. Mishin, M.J. Mehl, D. A. Papaconstantopoulos, A. F. Voter, J. D. Kress. Phys. Rev. B. 63224106 (2001).

4. Y. Mishin, M.J. Mehl and D. A. Papaconstantopoulos. Phys. Rev. B. 65, 224114 (2002).

5. R. R. Zope and Y. Mishin. Phys. Rev. B. 68, 024102 (2003).

6. A. Strachan, T. Cagin, O. Gu"lseren, S. Mukherjee, R. E. Cohen and W. A. Goddard. Model. Simul. Mater. Sci. Eng. 12, S445 (2004).
7. Y. Mishin, M.J. Mehl and D.A. Papaconstantopoulos. Acta Mater. 53, 4029 (2005).

8. Y. Mishin and A. Y. Lozovoi. Acta Mater. 54, (2006) 5013.

9. H. Chamati, N. Papanicolaou, Y. Mishin and D. A. Papaconstantopoulos. Surf. Sci. 600, 1793 (2006).

10. I. J. Robertson, V. Heine and M. C. Payne. Phys. Rev. Lett. 70, 1944 (1993).

11. S.L. Frederiksen, K.W. Jacobsen, K.S. Brown and J. P. Sethna. Phys. Rev. Lett. 93, 165501 (2004).

12. F. Cleri and V. Rosato. Phys. Rev. B. 48, 22 (1993).

13. E. F. Rexer, J. Jellinek, E.B. Krissinel, and E. K. Parks. J. Chem. Phys. 117, 82 (2002).

14. S. Darby, T. V. Mortimer-Jones, R.L. Johnston, and C. Roberts. J. Chem. Phys. 116, 1536 (2002).

15. K. Michaelian, M.R. Beltran, and I.L. Garzon. Phys. Rev. B. 65, 041403(R) (2002).

16. R.I. Babicheva, K.A. Bukreeva, S.V. Dmitriev, R.R. Mulyukov, K. Zhou, Intermetallics 43, (2013) 171.

17. K.A. Bukreeva, R.I. Babicheva, S.V. Dmitriev, K. Zhou, R.R. Mulyukov, JETP Lett. 98, (2013) 91.

18. R.I. Babicheva, K.A. Bukreeva, S.V. Dmitriev, K. Zhou, Computational Materials Science 79, (2013) 52.

19. K.A. Bukreeva, R.I. Babicheva, S.V. Dmitriev, K. Zhou, R.R. Mulyukov, Phys. Solid State 55, (2013) 1963.

20. M. Starostenkov, A. Yashin, N. Sinica. Key Engineering Materials. 592-593, 51 (2013).

21. M. M. Aish, M. D. Starostenkov. Mater.Phys.Mech. 18(1), 53 (2013).

22. M.D. Starostenkov, M.M. Aish, A.A. Sitnikov, S. A. Kotrechko. Letters on Mater. 3, 180 (2013).

23. K.A. Bukreeva, R.I. Babicheva, S.V. Dmitriev, K. Zhou, R.R. Mulyukov, A.I. Potekaev. Russian Physics Journal. 57(1), 69 (2014).

24. M. D. Starostenkov, M. M. Aish, A. V. Yashin. E-MRS 2013 FALL MEETING September 16-20. Warsaw University of Technology, Warsaw, Poland.

25. M.D. Starostenkov, M.M. Aish. 3rd International Conference on Mathematics \& Information Science (ICMIS 2013), Luxor, Egypt, Dec. 28-30, 2013.

26. M.M. Aish, M.D. Starostenkov. SOP Transactions on Nano-technology. (in press)

27. M.M. Aish, M.D. Starostenkov. E-MRS 2013 FALL MEETING September 16-20. Warsaw University of Technology, Warsaw, Poland. 